\title{
Adiponectin, markers of subclinical inflammation and nerve conduction in individuals with recently diagnosed type 1 and type 2 diabetes
}

\author{
Imke Schamarek 1,2,*, Christian Herder ${ }^{1,2, *}$, Bettina Nowotny ${ }^{1,2}$, \\ Maren Carstensen-Kirberg ${ }^{1,2}$, Klaus Straßburger 2,3, Peter Nowotny 1,2, \\ Alexander Strom ${ }^{1,2}$, Sonja Püttgen ${ }^{1,2}$, Karsten Müssig 1,2,4, Julia Szendroedi ${ }^{1,2,4}$, \\ Michael Roden ${ }^{1,2,4, * *}$ and Dan Ziegler ${ }^{1,2,4, * *}$ on behalf of the German Diabetes Study Group ${ }^{+}$
}

${ }^{1}$ Institute for Clinical Diabetology, German Diabetes Center, Leibniz Center for Diabetes Research at Heinrich Heine University Düsseldorf, Auf'm Hennekamp 65, 40225 Düsseldorf, Germany, ${ }^{2}$ German Center for Diabetes Research, München-Neuherberg, Germany, ${ }^{3}$ Institute for Biometrics and Epidemiology, German Diabetes Center, Leibniz Center for Diabetes Research at Heinrich Heine University Düsseldorf, Düsseldorf, Germany, and ${ }^{4}$ Department of Endocrinology and Diabetology, Medical Faculty, Heinrich Heine University Düsseldorf, Düsseldorf, Germany *(I Schamarek and C Herder share first authorship)

** (M Roden and D Ziegler share senior authorship)

${ }^{\dagger}$ (Details of the German Diabetes Study (GDS) group is presented in the Acknowledgements section)

\author{
Correspondence \\ should be addressed \\ to $\mathrm{C}$ Herder \\ Email \\ christian.herder@ \\ ddz.uni-duesseldorf.de
}

\begin{abstract}
Objective: Subclinical inflammation has been implicated in the development of diabetic sensorimotor polyneuropathy (DSPN), but studies using electrophysiological assessment as outcomes are scarce. Therefore, we aimed to investigate associations of biomarkers reflecting different aspects of subclinical inflammation with motor and sensory nerve conduction velocity (NCV) in individuals with diabetes.

Design and methods: Motor and sensory NCV was assessed in individuals with recently diagnosed type $2(n=352)$ or type 1 diabetes ( $n=161)$ from the baseline cohort of the observational German Diabetes Study. NCV sum scores were calculated for median, ulnar and peroneal motor as well as median, ulnar and sural sensory nerves. Associations between inflammationrelated biomarkers, DSPN and NCV sum scores were estimated using multiple regression models.

Results: In type 2 diabetes, high serum interleukin (IL)- 6 was associated with the presence of DSPN and reduced motor NCV. Moreover, higher levels of high-molecular weight (HMW) adiponectin, total adiponectin and their ratio were associated with prevalent DSPN and both diminished motor and sensory NCV, whereas no consistent associations were observed for C-reactive protein, IL18, soluble intercellular adhesion molecule-1 and E-selectin. In type 1 diabetes, only HMW and total adiponectin showed positive associations with motor NCV.

Conclusions: Our results point to a link between IL6 and both DSPN and slowed motor NCV in recently diagnosed type 2 diabetes. The reverse associations between adiponectin and NCV in type 1 and type 2 diabetes are intriguing, and further studies should explore whether they may reflect differences in the pathogenesis of DSPN in both diabetes types.
\end{abstract}

\section{Introduction}

Inflammatory processes have been implicated in the pathogenesis of experimental diabetic neuropathy (1). Subclinical inflammation, i.e. the form of low-grade inflammation that is characterised by elevated systemic levels of mainly proinflammatory immune mediators in the absence of clinical symptoms which is commonly (c) 2016 European Society of Endocrinology Printed in Great Britain
Published by Bioscientifica Ltd. 
found in patients with cardiometabolic diseases, has also been observed in diabetes patients with polyneuropathy. Several clinical studies demonstrated positive associations between systemic levels of proinflammatory cytokines and symptoms or signs of diabetic sensorimotor polyneuropathy (DSPN) in type 2 diabetes patients $(2,3,4,5,6,7,8)$ and in the older general population (9). Overall, observational findings are most consistent for interleukin (IL)-6 $(4,7,9)$, but there is also some evidence for associations of high-sensitivity C-reactive protein (hsCRP), proinflammatory cytokines and soluble cell adhesion molecules with diabetic neuropathy $(2,3,4,5,6,8)$.

In contrast, data regarding associations of adiponectin with DSPN are conflicting $(4,9,10,11,12)$. Adiponectin is an adipocyte-derived protein with multiple paracrine and endocrine activities. These include local and systemic anti-inflammatory, insulin-sensitising and proangiogenic effects so that a protective function in the context of diabetic polyneuropathy is conceivable $(13,14,15)$.

Only few studies investigated the association between biomarkers of subclinical inflammation and DSPN in type 1 diabetes. One study observed increased plasma levels of soluble tumour necrosis factor (TNF) receptors in patients with DSPN compared with patients without the condition, but no differences for hsCRP, IL6 or adiponectin (16). Another study reported that plasma sialic acid, a marker of the acute-phase response, was positively associated with diabetic neuropathy in men, but not independently of other risk factors (17).

However, the interpretation of the aforementioned studies is complicated by the fact that the definition of DSPN differed between studies. Definitions were based on varying combinations of subjective (neuropathic symptoms and signs) and objective (nerve conduction) measurements, therefore also reflecting different aspects of the pathophysiology of DSPN. Nerve conduction studies are important, because they give objective, noninvasive and highly reliable measures $(18,19)$. Abnormalities in nerve conduction appear to be among the first indications of DSPN in both type 1 and type 2 diabetes (20, 21). They are used to diagnose subclinical DSPN (22) and are of particular interest at early stages of diabetes as predictors of symptomatic DSPN, foot ulceration, amputation and mortality $(23,24,25)$. In addition, abnormal nerve conduction results are required to confirm the diagnosis of DSPN (22).

So far, data on the relationship between inflammation-related biomarkers and nerve conduction velocity (NCV) are scarce and mostly based on small study samples of patients with type 2 diabetes $(2,3,6,7,8,26)$.
Interestingly, one large population-based study indicated that circulating levels of the soluble IL6 receptor (sIL-6R) may be inversely associated with peroneal motor NCV (MNCV) (27).

The aim of this study was to test the hypothesis that serum levels of biomarkers reflecting different aspects of subclinical inflammation (acute-phase protein, proinflammatory cytokines, adiponectin, soluble adhesion molecules) are associated with DSPN based on the objective electrophysiological assessment and with the individual and summated motor and the sensory $\mathrm{NCV}$ in patients with recently diagnosed type 1 and type 2 diabetes.

\section{Subjects and methods}

\section{Study population}

The German Diabetes Study (GDS) is an ongoing prospective observational study, which evaluates the natural history of recent-onset diabetes and its sequelae (ClinicalTrials.gov identifier NTC01055093) $(21,28)$. The study is being conducted according to the declaration of Helsinki and was approved by the ethics committee of Heinrich Heine University, Düsseldorf, Germany. All participants provided written informed consent.

Inclusion criteria for entry into the GDS are type 1 or type 2 diabetes, time since diabetes diagnosis $\leq 1$ year and age of 18-69 years at the baseline examination. Diabetes was initially diagnosed by a general practitioner according to the guidelines of the American Diabetes Association (29). The concordance with these criteria was validated before inclusion into the study by the study centre staff. Exclusion criteria are secondary diabetes, current pregnancy, acute infections, cancer, evidence of congestive heart failure, kidney diseases, liver diseases, symptomatic peripheral arterial disease, psychiatric or addictive disorders, immunosuppressive therapy or participation in pharmacological intervention studies. The experimental design including a structured interview, anthropometry and measurement of HbA1c and lipids has been reported in detail before (28).

This cross-sectional analysis was based on consecutive participants who entered the study between its start in September 2005 and December $2011(n=513)$.

\section{Neurological examination and definition of DSPN}

Neurological examination was performed using the Neuropathy Disability Score (NDS) and the Neuropathy 
Symptom Score (NSS). Peripheral nerve function tests were performed as described before (21). Briefly, MNCV was determined in the median, ulnar and peroneal nerves, and sensory NCV (SNCV) as well as sensory nerve action potentials (SNAPs) were measured in the median, ulnar and sural nerves at a skin temperature of $33-34{ }^{\circ} \mathrm{C}$ with surface electrodes (Nicolet VikingQuest; Natus Medical, San Carlos, CA, USA).

Neuropathy stages were defined according to modified Toronto Consensus criteria (22). The definition of DSPN comprised subclinical DSPN (stage 1a: NDS $\leq 2$, NSS $\leq 2$, peroneal $\mathrm{MNCV}<2.5$ th percentile and sural $\mathrm{SNCV}<2.5$ th percentile and/or sural $\mathrm{SNAP}<2.5$ th percentile), confirmed asymptomatic DSPN (stage 1b: NDS $\geq 3$, $\mathrm{NSS} \leq 2$, peroneal $\mathrm{MNCV}<2.5$ th percentile and sural $\mathrm{SNCV}<2.5$ th percentile and/or sural SNAP $<2.5$ th percentile) and confirmed symptomatic DSPN (stage 2a: NSS $\geq 3$, peroneal $\mathrm{MNCV}<2.5$ th percentile and sural $\mathrm{SNCV}<2.5$ th percentile and/or sural SNAP $<2.5$ th percentile).

Only symptoms and signs were included in the clinical neuropathy scores that were considered by the experienced investigator to be due to distal sensorimotor polyneuropathy, but not other neurological diseases. Vitamin B6 or B12 deficiency as potential other causes of polyneuropathy cannot be completely ruled out, since their concentrations were not measured, but individuals with clinical symptoms or signs suspicious of manifest vitamin B6 or B12 deficiency were excluded from this study.

\section{Measurement of biomarkers of subclinical inflammation}

Serum concentrations of hsCRP, IL6, IL18, soluble intercellular adhesion molecule-1 (sICAM-1) and soluble E-selectin (sE-selectin) were measured as described before (28). Detailed information on biospecimen type, preanalytical processes and assay used for the quantification of total and high-molecular-weight (HMW) adiponectin is provided in Supplementary Table 1, see section on supplementary data given at the end of this article.

\section{Statistical analysis}

Analyses were stratified by diabetes type because there is evidence that risk factors of DSPN differ between type 1 and type 2 diabetes (30). Data are presented as mean \pm s.D., median (25th and 75th percentiles) or percentages (\%). Differences between diabetes types were tested using Student's $t$-test, Mann-Whitney test or $\chi^{2}$-test. Variables without normal distribution (triglycerides, all biomarkers of subclinical inflammation) were log-transformed before they entered regression models.

Associations between inflammation-related biomarkers and DSPN were assessed using multivariable logistic regression models with increasing complexity. Model 1 was adjusted for age and sex. Model 2 was additionally adjusted for time since diagnosis of diabetes, HbA1c, waist circumference, height, total cholesterol and hypertension. Model 3 adjusted for variables in model 2 and current smoking, physical activity, use of lipid-lowering medication, use of non-steroidal anti-inflammatory drugs and history of myocardial infarction and/or stroke. The latter variable was not used in the subgroup with type 1 diabetes, because only one individual was affected.

Associations between inflammation-related biomarkers and NCV as continuous variables were assessed using multivariable linear regression and the same covariables as in the logistic regression models. For the main analysis, MNCV and SNCV sum scores were calculated as the sums of individual z-scores of MNCV in the median, ulnar and peroneal nerves, and SNCV in the median, ulnar and sural nerves respectively. In both cases, the sum scores combine NCVs of three different nerves giving equal weight to each nerve and allowing a more comprehensive assessment of nerve function in an individual with low sum scores reflecting a more severe impairment of peripheral nerve function. In addition, the same analyses were performed for NCV and SNAPs in the individual nerves, which constitute the sum scores.

All statistical analyses were carried out using SPSS version 22 (IBM). A $P$ value of $<0.05$ was considered to indicate statistically significant differences or associations.

\section{Results}

\section{Study population}

Table 1 provides an overview of the study sample which included 352 individuals with type 2 diabetes and 161 individuals with type 1 diabetes. The subgroup with type 2 diabetes was characterised by a higher proportion of men, higher age, higher BMI and waist circumference, shorter height, better glycaemic control, higher lipid levels and blood pressure, less physical activity and higher prevalence of myocardial infarction and/or stroke compared with the subgroup of type 1 diabetes. Systemic levels of hsCRP, IL6, IL18 and soluble adhesion molecules were higher, total and HMW adiponectin levels lower in type 2 
Table 1 Patient characteristics stratified by diabetes type.

\begin{tabular}{l} 
Variable \\
\hline Sex (\% male) \\
Age (years) \\
BMI (kg/m²) \\
Waist circumference (cm) \\
Height (cm) \\
Time since diagnosis of diabetes (days) \\
HbA1c (\%) \\
HbA1c (mmol/mol) \\
Total cholesterol (mmol/l) \\
Fasting triglycerides (mmol/l) \\
Systolic blood pressure (mmHg) \\
Diastolic blood pressure (mmHg) \\
Hypertension (\%) \\
Current smoking (\%) \\
Physically inactive (\%) \\
History of myocardial infarction and/or stroke (\%) \\
Lipid-lowering medication (\%) \\
Antihypertensive medication (\%) \\
Non-steroidal anti-inflammatory drugs (\%) \\
Biomarkers of subclinical inflammation \\
hsCRP (mg/l) \\
IL6 (pg/ml) \\
IL18 (pg/ml) \\
Total adiponectin (ng/ml) \\
HMW adiponectin (ng/ml) \\
HMW/total adiponectin \\
sE-selectin (ng/ml) \\
sICAM-1 (ng/ml) \\
DSPN (\%) \\
NDS \\
NSS \\
Motor nerve conduction velocity (MNCV) \\
Median MNCV (m/s) \\
Ulnar MNCV (m/s) \\
Peroneal MNCV (m/s) \\
Sensory nerve conduction velocity (SNCV) \\
Median SNCV (m/s) \\
Ulnar SNCV (m/s) \\
Sural SNCV (m/s) \\
\end{tabular}

\begin{tabular}{c}
\hline Type 2 diabetes $(n=352)$ \\
\hline 66.2 \\
$52.6 \pm 10.6$ \\
$31.7 \pm 6.1$ \\
$106 \pm 14$ \\
$172 \pm 9$ \\
$181 \pm 96$ \\
$6.53 \pm 1.09$ \\
$48 \pm 12$ \\
$5.2 \pm 1.1$ \\
$1.4(1.0 ; 2.1)$ \\
$142 \pm 18$ \\
$85 \pm 11$ \\
60.1 \\
24.6 \\
39.9 \\
5.1 \\
23.6 \\
52.1 \\
4.5
\end{tabular}

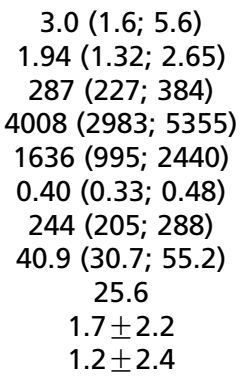

$53.4 \pm 3.2$

$55.8 \pm 5.7$

$44.7 \pm 5.1$

$51.7 \pm 6.8$

$53.3 \pm 5.8$

$44.6 \pm 5.8$

\begin{tabular}{|c|c|}
\hline Type 1 diabetes $(n=161)$ & $\boldsymbol{P}$ \\
\hline 60.9 & $<0.001$ \\
\hline $35.9 \pm 12.5$ & $<0.001$ \\
\hline $24.9 \pm 4.3$ & $<0.001$ \\
\hline $87 \pm 14$ & $<0.001$ \\
\hline $176 \pm 10$ & 0.001 \\
\hline $195 \pm 100$ & 0.125 \\
\hline $6.91 \pm 1.70$ & 0.008 \\
\hline $52 \pm 19$ & 0.008 \\
\hline $4.7 \pm 0.9$ & $<0.001$ \\
\hline $0.8(0.6 ; 1.2)$ & $<0.001$ \\
\hline $131 \pm 15$ & $<0.001$ \\
\hline $78 \pm 10$ & $<0.001$ \\
\hline 16.1 & $<0.001$ \\
\hline 25.6 & 0.82 \\
\hline 23.0 & $<0.001$ \\
\hline 0.6 & 0.010 \\
\hline 2.5 & $<0.001$ \\
\hline 8.1 & $<0.001$ \\
\hline 1.9 & 0.961 \\
\hline $1.1(0.6 ; 2.0)$ & $<0.001$ \\
\hline $0.88(0.65 ; 1.38)$ & $<0.001$ \\
\hline $260(183 ; 322)$ & $<0.001$ \\
\hline $6101(4112 ; 8095)$ & $<0.001$ \\
\hline $2751(1557 ; 4280)$ & $<0.001$ \\
\hline $0.45(0.36 ; 0.54)$ & 0.003 \\
\hline $217(182 ; 253)$ & $<0.001$ \\
\hline $34.5(24.8 ; 44.9)$ & $<0.001$ \\
\hline 19.3 & $<0.001$ \\
\hline $0.6+1.3$ & $<0.001$ \\
\hline $0.5 \pm 1.6$ & $<0.001$ \\
\hline $55.5 \pm 3.9$ & $<0.001$ \\
\hline $56.7 \pm 5.3$ & 0.097 \\
\hline $46.1 \pm 4.0$ & 0.002 \\
\hline $55.3 \pm 6.0$ & $<0.001$ \\
\hline $54.1 \pm 4.4$ & 0.100 \\
\hline $45.6 \pm 5.0$ & 0.066 \\
\hline
\end{tabular}

Data are given as mean \pm s.D., median (25th and 75th percentile) or \%. The maximum number of missing data is 28 for T2D and 7 for T1D. Hypertension is defined as blood pressure $\geq 140 / 90 \mathrm{mmHg}$ and/or use of antihypertensive medication.

'Physical inactivity is defined as absence of regular physical training at the time of the examination.

diabetes compared with type 1 diabetes. The prevalence of DSPN was higher among individuals with type 2 diabetes, which was mirrored by lower NCV.

\section{Association between biomarkers of subclinical inflammation and DSPN in type 2 and type 1 diabetes}

Among individuals with type 2 diabetes, IL6, total adiponectin and HMW adiponectin were positively associated with the presence of DSPN in age- and sexadjusted analyses (Table 2 , model 1 ). These associations remained significant in the fully adjusted model (Table 2 , model 3). In contrast, no associations were observed for
hsCRP, IL18, sE-selectin and sICAM-1. None of the measured biomarkers of subclinical inflammation displayed significant associations with DSPN among individuals with type 1 diabetes.

\section{Association between biomarkers of subclinical inflammation and NCV in type 2 diabetes}

In line with the aforementioned findings regarding DSPN, total adiponectin, HMW adiponectin and their ratio were inversely associated with sum scores of both MNCV and SNCV in the fully adjusted models (Table 3). In addition, IL6 levels were inversely associated with motor NCV, and 
Table 2 Association between biomarkers of subclinical inflammation and presence of DSPN.

\begin{tabular}{lll}
\hline Diabetes type & & Immune mediator \\
\cline { 1 - 2 } Type 2 diabetes & & hsCRP \\
& IL6 \\
& IL18 \\
& Total adiponectin \\
& HMW adiponectin \\
& HMW/total adiponectin \\
& SE-selectin \\
& sICAM-1 \\
Type 1 diabetes & hsCRP \\
& IL6 \\
& IL18 \\
& Total adiponectin \\
& HMW adiponectin \\
& HMW/total adiponectin \\
& sE-selectin \\
& sICAM-1 \\
&
\end{tabular}

\begin{tabular}{|c|c|}
\hline \multicolumn{2}{|c|}{ Model 1} \\
\hline$\beta$ & $P$ \\
\hline 0.207 & 0.136 \\
\hline 0.513 & 0.028 \\
\hline-0.282 & 0.352 \\
\hline 0.784 & 0.016 \\
\hline 0.478 & 0.021 \\
\hline 0.812 & 0.077 \\
\hline 0.241 & 0.416 \\
\hline 0.417 & 0.329 \\
\hline 0.197 & 0.327 \\
\hline 0.201 & 0.573 \\
\hline 0.025 & 0.953 \\
\hline-0.477 & 0.271 \\
\hline-0.333 & 0.248 \\
\hline-0.682 & 0.323 \\
\hline 0.135 & 0.778 \\
\hline 0.385 & 0.607 \\
\hline
\end{tabular}

\begin{tabular}{ccc}
\hline \multicolumn{2}{c}{ Model 2 } \\
\hline \multicolumn{1}{c}{$\beta$} & & $P$ \\
\cline { 1 - 1 } 0.179 & & 0.261 \\
0.486 & & 0.058 \\
-0.515 & & 0.114 \\
0.949 & & 0.006 \\
0.579 & & 0.005 \\
0.974 & & 0.045 \\
0.008 & & 0.980 \\
0.151 & & 0.734 \\
0.249 & & 0.301 \\
0.155 & & 0.707 \\
-0.003 & & 0.994 \\
-0.692 & & 0.166 \\
-0.457 & & 0.154 \\
-0.838 & & 0.248 \\
0.036 & & 0.945 \\
0.340 & & 0.675 \\
\hline
\end{tabular}

\begin{tabular}{|c|c|}
\hline \multicolumn{2}{|c|}{ Model 3} \\
\hline$\beta$ & $P$ \\
\hline 0.175 & 0.216 \\
\hline 0.575 & 0.039 \\
\hline-0.484 & 0.152 \\
\hline 1.007 & 0.005 \\
\hline 0.615 & 0.007 \\
\hline 1.057 & 0.032 \\
\hline 0.005 & 0.988 \\
\hline 0.149 & 0.760 \\
\hline 0.244 & 0.337 \\
\hline 0.118 & 0.796 \\
\hline-0.032 & 0.946 \\
\hline-0.883 & 0.097 \\
\hline-0.578 & 0.083 \\
\hline-1.086 & 0.142 \\
\hline 0.125 & 0.827 \\
\hline 0.879 & 0.386 \\
\hline
\end{tabular}

The table gives regression coefficients $(\beta)$ and corresponding $P$ values from logistic regression models. Immune mediators entered the models as logtransformed levels in the units given in Table 1. Model 1: adjusted for age and sex. Model 2: model 1+time since diagnosis of diabetes, HbA1c, waist circumference, height, total cholesterol, hypertension. Model 3: model 2+current smoking, physical activity, use of lipid-lowering medication, use of NSAIDs, history of myocardial infarction and/or stroke (the latter variable for type 2 diabetes only).

hsCRP levels were inversely associated with motor NCV in model 1, but the latter association failed to reach statistical significance after further adjustment (Table 3). In the fully adjusted model, IL18 was positively associated with sensory NCV.

The associations of total and HMW adiponectin with MNCV were mainly driven by peroneal NCV (Supplementary Table 2 , see section on supplementary data given at the end of this article), whereas their association with SNCV was similar for median, ulnar and sural SNCV (Supplementary Table 3). In addition, IL6 levels were inversely associated with median MNCV, and sICAM-1 levels were positively associated with median SNCV (Supplementary Tables 2 and 3).

There was also evidence for inverse associations of total and HMW adiponectin with median and sural SNAPs ( $P$ between 0.024 and 0.084 ), whereas IL6 was inversely associated with ulnar SNAP $(P=0.007)$ in the fully adjusted models (data not shown).

\section{Association between biomarkers of subclinical inflammation and NCV in type 1 diabetes}

In type 1 diabetes, hsCRP, IL6 and sICAM-1 levels were inversely associated with motor NCV. However, statistical significance was lost after further adjustment. In contrast, adjustment strengthened the positive associations between total and HMW adiponectin, which reached statistical significance in the full model. No associations were observed after full adjustment for sensory NCV (Table 4).

The associations of total and HMW adiponectin with motor NCV were strongest for ulnar and peroneal nerves. Some inverse associations between hsCRP, IL6 and sICAM1 were observed in median and ulnar nerves, but not in the full model (Supplementary Table 4, see section on supplementary data given at the end of this article). Inverse associations between HMW adiponectin and the ratio of HMW to total adiponectin with ulnar SNCV were the only significant associations with sensory NCV after full adjustment (Supplementary Table 5).

Neither total nor HMW adiponectin levels were associated with median, ulnar or sural SNAPs (data not shown).

\section{Discussion}

This study identified associations between multiple biomarkers of subclinical inflammation and NCV in patients with recently diagnosed type 2 or type 1 diabetes by three main findings: i) in individuals with type 2 diabetes, high serum IL6 was associated with the presence of DSPN and reduced motor NCV, whereas no consistent associations were observed for hsCRP, IL18, sICAM-1 and E-selectin; ii) higher levels of HMW and total adiponectin were consistently associated with the presence of DSPN and both reduced motor and sensory NCV in individuals with type 2 diabetes; and iii) by 
Table 3 Association between biomarkers of subclinical inflammation and sum scores for nerve conduction velocity (NCV) in patients with type 2 diabetes.

\begin{tabular}{|c|c|c|c|c|c|c|}
\hline \multirow[b]{2}{*}{ Immune mediator } & \multicolumn{2}{|c|}{ Model 1} & \multicolumn{2}{|c|}{ Model 2} & \multicolumn{2}{|c|}{ Model 3} \\
\hline & $\beta$ & $P$ & $\beta$ & $P$ & $\beta$ & $P$ \\
\hline \multicolumn{7}{|l|}{ Motor NCV } \\
\hline hsCRP & -0.128 & 0.019 & -0.097 & 0.107 & -0.109 & 0.080 \\
\hline IL6 & -0.184 & 0.001 & -0.152 & 0.005 & -0.161 & 0.006 \\
\hline IL18 & 0.032 & 0.531 & 0.081 & 0.117 & 0.088 & 0.091 \\
\hline Total adiponectin & -0.060 & 0.289 & -0.094 & 0.088 & -0.111 & 0.048 \\
\hline HMW adiponectin & -0.054 & 0.339 & -0.096 & 0.083 & -0.117 & 0.037 \\
\hline HMW/total adiponectin & -0.035 & 0.517 & -0.079 & 0.141 & -0.103 & 0.058 \\
\hline sE-selectin & -0.032 & 0.530 & 0.028 & 0.604 & 0.045 & 0.406 \\
\hline sICAM-1 & -0.067 & 0.197 & -0.023 & 0.652 & 0.001 & 0.985 \\
\hline \multicolumn{7}{|l|}{ Sensory NCV } \\
\hline hsCRP & -0.047 & 0.389 & -0.019 & 0.758 & -0.026 & 0.689 \\
\hline IL6 & -0.062 & 0.236 & -0.036 & 0.524 & -0.034 & 0.575 \\
\hline IL18 & 0.075 & 0.146 & 0.103 & 0.0496 & 0.110 & 0.040 \\
\hline Total adiponectin & -0.163 & 0.004 & -0.180 & 0.001 & -0.193 & 0.001 \\
\hline HMW adiponectin & -0.160 & 0.004 & -0.180 & 0.001 & -0.196 & 0.001 \\
\hline HMW/total adiponectin & -0.126 & 0.021 & -0.143 & 0.009 & -0.162 & 0.003 \\
\hline sE-selectin & -0.066 & 0.201 & -0.017 & 0.753 & -0.010 & 0.863 \\
\hline sICAM-1 & -0.003 & 0.954 & 0.027 & 0.610 & 0.038 & 0.509 \\
\hline
\end{tabular}

The table gives regression coefficients $(\beta)$ and corresponding $P$ values from linear regression models. Immune mediators entered the models as logtransformed levels in the units given in Table 1. Model 1, adjusted for age and sex; Model 2, model 1+time since diagnosis of diabetes, HbA1c, waist circumference, height, total cholesterol, hypertension; Model 3, model 2+current smoking, physical activity, use of lipid-lowering medication, use of NSAIDs, history of myocardial infarction and/or stroke.

contrast, in participants with type 1 diabetes, associations between high HMW and total adiponectin and higher motor NCV were found.

\section{Subclinical inflammation, DSPN and NCV in type 2 diabetes}

This study extends the current literature as it represents a comprehensive analysis of associations between biomarkers reflecting different aspects of subclinical inflammation and NCV in a fairly large study sample of patients with recently diagnosed diabetes. Another novel aspect of the study is the calculation of sum scores in the main analysis rather than analysing single nerves. This approach was chosen following the recommendation to use composite nerve conduction test scores to identify early nerve dysfunction in diabetes (24). Different composite scores have been validated by other studies investigating associations between NCV and future DSPN $(24,25)$ as well as associations between inflammation and NCV (8).

Our first main finding regarding associations of higher serum IL6 with reduced MNCV, but not with SNCV, suggests a differential risk conferred by IL6 for NCV slowing between motor and sensory nerves. These results are of particular interest in the context of data from InCHIANTI, a population-based study in Italy, which reported associations of higher IL6 and sIL-6R levels with reduced peroneal MNCV (27). Furthermore, an association of higher IL6 with reduced sural and peroneal NAPs was observed in a small study (7). Collectively, these studies underline the notion that the IL6 system may represent a determinant of motor nerve function and integrity.

In contrast, we did not find consistent associations between hsCRP and DSPN or NCV. Data regarding the relationship of hsCRP with DSPN have been less consistent $(4,5,9)$ than the association between IL6 and DSPN. Overall, these data are reminiscent of the role of inflammation in the development of cardiovascular disease (CVD). IL6 is one of the main inducers of CRP, and even if hsCRP is used frequently as risk marker for CVD, there is considerably more evidence for a causal role of the IL6 system in the development of CVD (31), which itself represents a risk factor for DSPN.

Our second main finding of robust associations between higher total adiponectin, higher HMW adiponectin and higher values of their ratio (HMW/total) on the one hand and the presence of DSPN and reduced NCV on the other hand may appear counterintuitive, because adiponectin shows consistent associations with lower risk of diabetes in humans and because of its antiatherogenic properties in preclinical studies $(13,15)$. Our observation may appear particularly surprising given that it has been 
Table 4 Association between biomarkers of subclinical inflammation and sum scores for nerve conduction velocity (NCV) in patients with type 1 diabetes.

\begin{tabular}{|c|c|c|c|c|c|c|c|}
\hline \multirow[b]{2}{*}{ Nerve type } & \multirow[b]{2}{*}{ Immune mediator } & \multicolumn{2}{|c|}{ Model 1} & \multicolumn{2}{|c|}{ Model 2} & \multicolumn{2}{|c|}{ Model 3} \\
\hline & & $\beta$ & $P$ & $\beta$ & $P$ & $\beta$ & $P$ \\
\hline \multirow[t]{8}{*}{ Motor NCV } & hsCRP & -0.176 & 0.027 & -0.162 & 0.073 & -0.107 & 0.238 \\
\hline & IL6 & -0.233 & 0.003 & -0.209 & 0.017 & -0.120 & 0.199 \\
\hline & IL18 & -0.014 & 0.859 & 0.005 & 0.954 & 0.017 & 0.830 \\
\hline & Total adiponectin & 0.112 & 0.188 & 0.143 & 0.113 & 0.211 & 0.017 \\
\hline & HMW adiponectin & 0.113 & 0.198 & 0.133 & 0.141 & 0.208 & 0.019 \\
\hline & HMW/total adiponectin & 0.085 & 0.326 & 0.087 & 0.314 & 0.154 & 0.072 \\
\hline & sE-selectin & -0.012 & 0.878 & 0.043 & 0.606 & 0.088 & 0.298 \\
\hline & sICAM-1 & -0.179 & 0.020 & -0.180 & 0.023 & -0.117 & 0.195 \\
\hline \multirow[t]{8}{*}{ Sensory NCV } & hsCRP & -0.088 & 0.264 & -0.131 & 0.152 & -0.141 & 0.139 \\
\hline & IL6 & 0.035 & 0.650 & 0.015 & 0.866 & -0.001 & 0.991 \\
\hline & IL18 & 0.028 & 0.721 & 0.005 & 0.951 & 0.010 & 0.900 \\
\hline & Total adiponectin & -0.078 & 0.353 & -0.055 & 0.541 & -0.045 & 0.631 \\
\hline & HMW adiponectin & -0.132 & 0.126 & -0.111 & 0.221 & -0.101 & 0.280 \\
\hline & HMW/total adiponectin & -0.187 & 0.028 & -0.165 & 0.055 & -0.160 & 0.075 \\
\hline & sE-selectin & -0.015 & 0.850 & -0.024 & 0.771 & -0.008 & 0.928 \\
\hline & sICAM-1 & -0.078 & 0.313 & -0.116 & 0.150 & -0.146 & 0.118 \\
\hline
\end{tabular}

The table gives regression coefficients $(\beta)$ and corresponding $P$ values from linear regression models. Immune mediators entered the models as logtransformed levels in the units given in Table 1. Model 1: adjusted for age and sex. Model 2: model 1+time since diagnosis of diabetes, HbA1c, waist circumference, height, total cholesterol, hypertension. Model 3: model 2+current smoking, physical activity, use of lipid-lowering medication and use of NSAIDs.

hypothesised that HMW adiponectin and a higher $\mathrm{HMW} /$ total adiponectin ratio may be even more strongly associated with favourable cardiometabolic health than total adiponectin (32).

We are not aware of studies that have demonstrated an independent inverse association between circulating adiponectin (irrespective of its isoforms) and DSPN. Whereas no associations were found in some populations $(4,9,10)$, others also observed increased levels of total adiponectin in DSPN $(11,12)$. However, our study extends these data because we i) included measurements of HMW adiponectin, ii) performed more extensive adjustment to rule out false-positive findings due to confounding and iii) provide novel data on the associations between adiponectin and both MNCV and SNCV. It is interesting to note that the association with $\mathrm{MNCV}$ was strongest for the peroneal nerve, which is often used as a surrogate endpoint in clinical studies and to monitor progression of DSPN.

Since the literature on adiponectin and DSPN is scarce, one may compare the present study with studies investigating associations between adiponectin and other microvascular outcomes. Indeed, higher levels of total adiponectin have been found in patients with type 2 diabetes and retinopathy compared with those without retinopathy $(10,11,12)$. In addition, inverse or no associations of total adiponectin have been reported with respect to cardiovascular risk in the general population, whereas positive associations were found in patients with type 2 diabetes and/or micro and macrovascular disease $(33,34)$. HMW adiponectin levels were inversely associated with cardiac autonomic imbalance in type 2 diabetes in one study (35), while a second study reported a positive association between total adiponectin and cardiac autonomic neuropathy (36), underlining the need for further investigations.

From a mechanistic perspective, we hypothesised that proinflammatory immune mediators would be associated with reduced NCV reflecting impaired integrity and degree of myelination of large fibres (18). It is biologically plausible that elevated circulating levels of IL6, which has various proinflammatory properties, may cause structural and functional deficits of nerve fibres by triggering intracellular signaling downstream of the IL6R/gp130 complex or by indirect effects through activation of macrophages and other cell types.

In contrast, adiponectin has anti-inflammatory properties (13), and currently there are no data supporting a direct detrimental impact of adiponectin on nerve function. It has been suggested that adiponectin could be upregulated by metabolic and/or inflammatory insults, which are causal for the development of adverse cardiometabolic outcomes so that adiponectin may be interpreted as indirect risk marker, not as genuine risk factor (37). With respect to cardiovascular risk, adiponectin expression and release is increased by atrial natriuretic 
peptide (ANP) and brain natriuretic peptide (BNP) (38). In order to rule out confounding by CVD, we adjusted for the history of myocardial infarction and/or stroke and for multiple cardiovascular risk factors. However, even if the mechanism linking adiponectin and DSPN is not clear yet, our data strongly suggest that although adiponectin may serve as protective factor for incident diabetes, the time around the manifestation of type 2 diabetes may be characterised by metabolic and/or proinflammatory perturbations that reverse the direction of the association between adiponectin levels and risk of micro and macrovascular complications.

\section{Subclinical inflammation, DSPN and NCV in type 1 diabetes}

This study indicates that there are both similarities and differences in associations between biomarkers of subclinical inflammation and NCV between type 1 and type 2 diabetes. In both diabetes types we found inverse associations between high hsCRP and IL6 on the one hand and reduced motor NCV on the other hand in our initial models. Effect estimates in the final models were similar, but adjustment for potential confounders attenuated the associations in type 1 diabetes to non-significance, which most likely reflects reduced statistical power in this subgroup. The lack of associations between IL6 and sensory NCV as well as the absence of associations between soluble adhesion molecules and NCV in the final models were also comparable between both diabetes types.

Clear differences were observed for adiponectin, which was inversely associated with both MNCV and SNCV in type 2 diabetes, whereas a positive association was found with MNCV in type 1 diabetes. It is well known that patients with type 1 diabetes have increased levels of total and HMW adiponectin in the circulation, whereas decreased levels are found in type 2 diabetes $(39,40)$. The mechanism behind this upregulation and its physiological relevance are currently only poorly understood. Although adiponectin levels in type 1 diabetes are also positively associated with insulin sensitivity, insulin sensitivity is lower at any given adiponectin level compared with nondiabetic controls (41). Prospective studies demonstrated that higher adiponectin levels in type 1 diabetes are linked to an elevated risk of progression of nephropathy and increased cardiovascular and all-cause mortality $(42,43)$. The difference in adiponectin between both diabetes types may be due to the interplay of insulin and adiponectin in a way that adiponectin levels are increased in type 1 diabetes due to the lack of endogenous insulin production, whereas they are decreased in response to hyperinsulinaemia in type 2 diabetes $(44,45)$. Alternative explanations implicate increased adiponectin levels as reflection of underlying CVD or impaired renal function (45), but these mechanisms would apply to both diabetes types and are unlikely to play a role in our cohort of patients with recently diagnosed diabetes.

Adiponectin levels are also determined by genetic variants within the gene encoding adiponectin and in other loci, and these effects may be modified by dietary components (46). Although one small study indicated that polymorphisms within the adiponectin gene may be associated with polyneuropathy in type 2 diabetes (47), there is currently insufficient evidence for the hypothesis that different gene variants that have an impact on adiponectin levels could explain the differences in adiponectin levels and their associations with diabetic complications between type 1 and type 2 diabetes.

It is currently not possible to infer whether the upregulation of adiponectin in type 1 diabetes reflects a protective mechanism to maintain motor nerve function or whether this association is confounded. Our data support the hypothesis that the pathogenesis of DSPN may differ between type 1 and type 2 diabetes and involve at least in part different sets of risk factors with a more prominent role of metabolic risk factors and subclinical inflammation in type 2 diabetes compared with type 1 diabetes (30).

\section{Strengths and limitations}

The comparably short known duration of disease represents a strength of the study, because it allowed to analyse associations between inflammatory markers and DSPN before the onset of potentially confounding diabetes-related complications. Detailed nerve conduction studies for assessing large-fibre function were carried out rigorously using gold-standard methods, validated criteria and reference values. Our statistical analysis included sum scores for MNCV and SNCV rather than NCV of single nerves and adjusted for multiple confounders.

The limitations include the cross-sectional design precluding the assessment of temporal relationships between the upregulation of inflammation-related biomarkers and changes in NCV. We could not assess the direct impact of glucose-lowering treatment on inflammation-related biomarkers, because blood samples from before the onset of therapy were not available. The statistical power differed between both diabetes types as 
a consequence of the study design (inclusion of consecutive study participants with skewed enrolment regarding diabetes types), which entails the risk of type II errors in the smaller subsample of individuals with type 1 diabetes. Finally, study population of adult patients with short known diabetes duration and rather good glycaemic control renders our data not generalisable to diabetes patients with longer disease duration and/or worse glycaemic control.

\section{Conclusions}

This study demonstrates that increased serum IL6 is associated with reduced NCV in type 2 diabetes and corroborates the hypothesis that the IL6 system may contribute to peripheral large nerve fibre dysfunction and DSPN. Circulating total and HMW adiponectin are also associated with NCV in type 2 and type 1 diabetes, but in opposite directions. The association between high adiponectin and reduced NCV is consistent with positive associations between adiponectin and retinopathy or cardiovascular outcomes in type 2 diabetes. Our data support the notion that the pathomechanisms leading to DSPN may only partially overlap between type 1 and type 2 diabetes.

Supplementary data

This is linked to the online version of the paper at http://dx.doi.org/10.1530/ EJE-15-1010.

Declaration of interest

The authors declare that there is no conflict of interest that could be perceived as prejudicing the impartiality of the research reported.

\section{Funding}

The German Diabetes Study was initiated and is performed by the DDZ, which is funded by the German Federal Ministry of Health and the Ministry of Innovation, Science, Research and Technology of the State North RhineWestphalia. This study was supported in part by a grant from the German Federal Ministry of Education and Research (BMBF) to the German Center for Diabetes Research (DZD e.V.) and by a grant of the Helmholtz Alliance Imaging and Curing Environmental Metabolic Diseases (ICEMED). The funders had no role in study design, data collection and analysis, decision to publish or preparation of the manuscript.

\section{Author contribution statement}

I Schamarek designed the study, performed the statistical analysis, interpreted the data, contributed to discussion and critically reviewed the manuscript. C Herder designed the study, researched data, contributed to data analysis, interpreted the data and wrote the manuscript.
B Nowotny researched data and critically reviewed the manuscript. M Carstensen-Kirberg researched data, contributed to discussion and critically reviewed the manuscript. $\mathrm{K}$ Straßburger contributed to data analysis, contributed to discussion and critically reviewed the manuscript. P Nowotny, A Strom and S Püttgen researched data and critically reviewed the manuscript. K Müssig and J Szendroedi researched data, contributed to discussion and critically reviewed the manuscript. M Roden designed the study, contributed to discussion, critically reviewed and edited the manuscript. D Ziegler designed the study, researched data, interpreted the data, contributed to discussion, critically reviewed and edited the manuscript. All authors approved the final version of the manuscript.

\section{Acknowledgements}

The authors appreciate the voluntary contribution of all study participants. They also thank the staff of the Clinical Research Center of the German Diabetes Center (DDZ, Düsseldorf, Germany) for excellent technical assistance and taking care of the patients. The authors further thank Ulrike Partke, Gabi Gornitzka, Margret Behler, and Maria Schroers-Teuber (DDZ) for excellent technical assistance.

GDS Group: M Roden (speaker), A E Buyken, J Eckel, G Geerling, H Al-Hasani, C Herder, A Icks, J Kotzka, O Kuss, E Lammert, J Lundbom, K Müssig, P Nowotny, W Rathmann, J Szendroedi, D Ziegler and their coworkers who are responsible for the design and performance of the GDS.

\section{References}

1 Zychowska M, Rojewska E, Przewlocka B \& Mika J. Mechanisms and pharmacology of diabetic neuropathy - experimental and clinical studies. Pharmacological Reports 201365 1601-1610. (doi:10.1016/ S1734-1140(13)71521-4)

2 Jude EB, Abbott CA, Young MJ, Anderson SG, Douglas JT \& Boulton AJ. The potential role of cell adhesion molecules in the pathogenesis of diabetic neuropathy. Diabetologia 199841 330-336. (doi:10.1007/ s001250050911)

3 Matsuda M, Kawasaki F, Inoue H, Kanda Y, Yamada K, Harada Y, Saito M, Eto M, Matsuki M \& Kaku K. Possible contribution of adipocytokines on diabetic neuropathy. Diabetes Research and Clinical Practice 200466 S121-S123. (doi:10.1016/j.diabres.2004.05.010)

4 Herder C, Lankisch M, Ziegler D, Rathmann W, Koenig W, Illig T, Döring A, Thorand B, Holle R, Giani G et al. Subclinical inflammation and diabetic polyneuropathy: MONICA/KORA Survey F3 (Augsburg, Germany). Diabetes Care 200932 680-682. (doi:10.2337/dc08-2011)

5 Doupis J, Lyons TE, Wu S, Gnardellis C, Dinh T \& Veves A. Microvascular reactivity and inflammatory cytokines in painful and painless peripheral diabetic neuropathy. Journal of Clinical Endocrinology \& Metabolism 200994 2157-2163. (doi:10.1210/jc.2008-2385)

6 Hussain G, Rizvi SA, Singhal S, Zubair M \& Ahmad J. Serum levels of $\mathrm{TNF}-\alpha$ in peripheral neuropathy patients and its correlation with nerve conduction velocity in type 2 diabetes mellitus. Diabetes \& Metabolic Syndrome: Clinical Research \& Reviews 20137 238-242. (doi:10.1016/ j.dsx.2013.02.005)

7 Magrinelli F, Briani C, Romano M, Ruggero S, Toffanin E, Triolo G, Peter GC, Praitano M, Lauriola MF, Zanette G et al. The association between serum cytokines and damage to large and small nerve fibers in diabetic peripheral neuropathy. Journal of Diabetes Research 20152015 547834. (doi:10.1155/2015/547834)

8 Kallestrup M, Møller HJ, Tankisi H \& Andersen H. Soluble CD163 levels are elevated in cerebrospinal fluid and serum in people with type 2 diabetes mellitus and are associated with impaired peripheral nerve function. Diabetic Medicine 201532 54-61. (doi:10.1111/dme.12568)

9 Herder C, Bongaerts BW, Rathmann W, Heier M, Kowall B, Koenig W, Thorand B, Roden M, Meisinger C \& Ziegler D. Association of 
subclinical inflammation with polyneuropathy in the older population: KORA F4 study. Diabetes Care 201336 3663-3670. (doi:10.2337/dc13-0382)

10 Kato K, Osawa H, Ochi M, Kusunoki Y, Ebisui O, Ohno K, Ohashi J, Shimizu I, Fujii Y, Tanimoto $\mathrm{M}$ et al. Serum total and high molecular weight adiponectin levels are correlated with the severity of diabetic retinopathy and nephropathy. Clinical Endocrinology 200868 442-449. (doi:10.1111/j.1365-2265.2007.03063.x)

11 Jung CH, Kim BY, Mok JO, Kang SK \& Kim CH. Association between serum adipocytokine levels and microangiopathies in patients with type 2 diabetes mellitus. Journal of Diabetes Investigation 20145 333-339. (doi:10.1111/jdi.12144)

12 Pradeepa R, Surendar J, Indulekha K, Chella S, Anjana RM \& Mohan V. Association of serum adiponectin with diabetic microvascular complications among south Indian type 2 diabetic subjects - (CURES-133). Clinical Biochemistry 201548 33-38. (doi:10.1016/j.clinbiochem.2014.10.009)

13 Turer AT \& Scherer PE. Adiponectin: mechanistic insights and clinical implications. Diabetologia 201255 2319-2326. (doi:10.1007/ s00125-012-2598-x)

14 Ye R \& Scherer PE. Adiponectin, driver or passenger on the road to insulin sensitivity? Molecular Metabolism 20132 133-141. (doi:10.1016/ j.molmet.2013.04.001)

15 Herder C, Carstensen M \& Ouwens DM. Anti-inflammatory cytokines and risk of type 2 diabetes. Diabetes Obesity \& Metabolism 201315 (Suppl 3) 39-50. (doi:10.1111/dom.12155)

16 González-Clemente JM, Mauricio D, Richart C, Broch M, Caixàs A, Megia A, Giménez-Palop O, Simón I, Martínez-Riquelme A, Giménez-Pérez G et al. Diabetic neuropathy is associated with activation of the TNF-alpha system in subjects with type 1 diabetes mellitus. Clinical Endocrinology 200563 525-529. (doi:10.1111/ j.1365-2265.2005.02376.x)

17 Soedamah-Muthu SS, Chaturvedi N, Witte DR, Stevens LK, Porta M, Fuller JH \& EURODIAB Prospective Complications Study Group . Relationship between risk factors and mortality in type 1 diabetic patients in Europe: the EURODIAB Prospective Complications Study (PCS). Diabetes Care 200831 1360-1366. (doi:10.2337/dc08-0107)

18 Boulton AJ, Malik RA, Arezzo JC \& Sosenko JM. Diabetic somatic neuropathies. Diabetes Care 200427 1458-1486. (doi:10.2337/diacare. 27.6.1458)

19 Dyck PJ, Herrmann DN, Staff NP \& Dyck PJ. Assessing decreased sensation and increased sensory phenomena in diabetic polyneuropathies. Diabetes 201362 3677-3686. (doi:10.2337/db13-0352)

20 Ziegler D, Behler M, Schroers-Teuber M \& Roden M. Near-normoglycaemia and development of neuropathy: a 24-year prospective study from diagnosis of type 1 diabetes. BMJ Open $2015 \mathbf{5}$ e006559. (doi:10.1136/bmjopen-2014-006559)

21 Ziegler D, Papanas N, Zhivov A, Allgeier S, Winter K, Ziegler I, Brüggemann J, Strom A, Peschel S, Köhler B et al. Early detection of nerve fiber loss by corneal confocal microscopy and skin biopsy in recently diagnosed type 2 diabetes. Diabetes $2014632454-2463$. (doi:10.2337/db13-1819)

22 Dyck PJ, Albers JW, Andersen H, Arezzo JC, Biessels GJ, Bril V, Feldman EL, Litchy WJ, O’Brien PC, Russell JW et al. Diabetic polyneuropathies: update on research definition, diagnostic criteria and estimation of severity. Diabetes Metabolism Research \& Reviews 2011 27 620-628. (doi:10.1002/dmrr.1226)

23 Carrington AL, Shaw JE, Van Schie CH, Abbott CA, Vileikyte L \& Boulton AJ. Can motor nerve conduction velocity predict foot problems in diabetic subjects over a 6-year outcome period? Diabetes Care 200225 2010-2015. (doi:10.2337/diacare.25.11.2010)

24 Dyck PJ, O’Brien PC, Litchy WJ, Harper CM, Klein CJ \& Dyck PJ. Monotonicity of nerve tests in diabetes: subclinical nerve dysfunction precedes diagnosis of polyneuropathy. Diabetes Care 200528 2192-2200. (doi:10.2337/diacare.28.9.2192)

25 Weisman A, Bril V, Ngo M, Lovblom LE, Halpern EM, Orszag A \& Perkins BA. Identification and prediction of diabetic sensorimotor polyneuropathy using individual and simple combinations of nerve conduction study parameters. PLoS ONE 20138 e58783. (doi:10.1371/ journal.pone.0058783)

26 Duksal T, Tiftikcioglu BI, Bilgin S, Kose S \& Zorlu Y. Role of inflammation in sensory neuropathy in prediabetes or diabetes. Acta Neurologica Scandinavica, 2015. In press. (doi:10.1111/ane.12474)

27 Di Iorio A, Cherubini A, Volpato S, Sparvieri E, Lauretani F, Franceschi C, Senin U, Abate G, Paganelli R, Martin A et al. Markers of inflammation, vitamin $\mathrm{E}$ and peripheral nervous system function: the InCHIANTI study. Neurobiology of Aging 200627 1280-1288. (doi:10.1016/j.neurobiolaging.2005.07.004)

28 Weber KS, Nowotny B, Strassburger K, Pacini G, Müssig K, Szendroedi J, Herder C, Roden M \& GDS Group . The role of markers of low-grade inflammation for the early time course of glycemic control, glucose disappearance rate, and $\beta$-cell function in recently diagnosed type 1 and type 2 diabetes. Diabetes Care 201538 1758-1767. (doi:10.2337/ dc15-0169)

29 American Diabetes Association. Diagnosis and classification of diabetes mellitus. Diabetes Care 200427 (Suppl 1) S5-S10. (doi:10.2337/diacare. 27.2007.S5)

30 Callaghan BC, Hur J \& Feldman EL. Diabetic neuropathy: one disease or two? Current Opinion in Neurology 201225 536-541. (doi:10.1097/WCO. Ob013e328357a797)

31 Interleukin-6 Receptor Mendelian Randomisation Analysis (IL6R MR) Consortium. The interleukin- 6 receptor as a target for prevention of coronary heart disease: a mendelian randomisation analysis. Lancet 2012379 1214-1224. (doi:10.1016/S0140-6736(12)60110-X)

32 Hara K, Horikoshi M, Yamauchi T, Yago H, Miyazaki O, Ebinuma H, Imai Y, Nagai R \& Kadowaki T. Measurement of the high-molecular weight form of adiponectin in plasma is useful for the prediction of insulin resistance and metabolic syndrome. Diabetes Care 200629 1357-1362. (doi:10.2337/dc05-1801)

33 Schöttker B, Herder C, Rothenbacher D, Roden M, Kolb H, Müller H \& Brenner H. Proinflammatory cytokines, adiponectin, and increased risk of primary cardiovascular events in diabetic patients with or without renal dysfunction: results from the ESTHER study. Diabetes Care 2013 36 1703-1711. (doi:10.2337/dc12-1416)

$34 \mathrm{Wu}$ ZJ, Cheng YJ, Gu WJ \& Aung LH. Adiponectin is associated with increased mortality in patients with already established cardiovascular disease: a systematic review and meta-analysis. Metabolism 201463 1157-1166. (doi:10.1016/j.metabol.2014.05.001)

35 Lieb DC, Parson HK, Mamikunian G \& Vinik AI. Cardiac autonomic imbalance in newly diagnosed and established diabetes is associated with markers of adipose tissue inflammation. Experimental Diabetes Research 20122012 878760. (doi:10.1155/2012/878760)

36 Jung CH, Kim BY, Kim CH, Kang SK, Jung SH \& Mok JO. Association of serum adipocytokine levels with cardiac autonomic neuropathy in type 2 diabetic patients. Cardiovascular Diabetology 20121124. (doi:10.1186/1475-2840-11-24)

37 Rathmann W \& Herder C. Adiponectin and cardiovascular mortality: evidence for "reverse epidemiology". Hormone and Metabolic Research 200739 1-2. (doi:10.1055/s-2007-958630)

38 Tsukamoto O, Fujita M, Kato M, Yamazaki S, Asano Y, Ogai A, Okazaki H, Asai M, Nagamachi Y, Maeda N et al. Natriuretic peptides enhance the production of adiponectin in human adipocytes and in patients with chronic heart failure. Journal of the American College of Cardiology 200953 2070-2077. (doi:10.1016/j.jacc.2009.02.038)

39 Imagawa A, Funahashi T, Nakamura T, Moriwaki M, Tanaka S, Nishizawa H, Sayama K, Uno S, Iwahashi H, Yamagata K et al. Elevated serum concentration of adipose-derived factor, adiponectin, in patients with type 1 diabetes. Diabetes Care 200225 1665-1666. (doi:10.2337/ diacare.25.9.1665)

40 Leth H, Andersen KK, Frystyk J, Tarnow L, Rossing P, Parving HH \& Flyvbjerg A. Elevated levels of high-molecular-weight adiponectin in type 1 diabetes. Journal of Clinical Endocrinology \& Metabolism 200893 3186-3191. (doi:10.1210/jc.2008-0360) 
41 Pereira RI, Snell-Bergeon JK, Erickson C, Schauer IE, Bergman BC, Rewers M \& Maahs DM. Adiponectin dysregulation and insulin resistance in type 1 diabetes. Journal of Clinical Endocrinology \& Metabolism 201297 E642-E647. (doi:10.1210/ jc.2011-2542)

42 Saraheimo M, Forsblom C, Thorn L, Wadén J, Rosengård-Bärlund M, Heikkilä O, Hietala K, Gordin D, Frystyk J, Flyvbjerg A et al. Serum adiponectin and progression of diabetic nephropathy in patients with type 1 diabetes. Diabetes Care 200831 1165-1169. (doi:10.2337/dc07-2306)

43 Forsblom C, Thomas MC, Moran J, Saraheimo M, Thorn L, Wadén J, Gordin D, Frystyk J, Flyvbjerg A, Groop PH et al. Serum adiponectin concentration is a positive predictor of all-cause and cardiovascular mortality in type 1 diabetes. Journal of Internal Medicine 2011270 346-355. (doi:10.1111/j.1365-2796.2011. 02406.x)
44 Cook JR \& Semple RK. Hypoadiponectinemia - cause or consequence of human "insulin resistance"? Journal of Clinical Endocrinology \& Metabolism 201095 1544-1554. (doi:10.1210/jc.2009-2286)

45 Sattar N. Adiponectin and raised mortality in type 1 diabetes: any credible explanatory mechanisms? Journal of Internal Medicine 2011270 339-342. (doi:10.1111/j.1365-2796.2011.02408.x)

46 Hwang JY, Park JE, Choi YJ, Huh KB, Chang N \& Kim WY. Carbohydrate intake interacts with SNP276G $>$ T polymorphism in the adiponectin gene to affect fasting blood glucose, $\mathrm{HbA1C}$, and HDL cholesterol in Korean patients with type 2 diabetes. Journal of the American College of Nutrition 201332 143-150.

47 Ji ZY, Li HF, Lei Y, Rao YW, Tan ZX, Liu HJ, Yao GD, Hou B \& Sun ML. Association of adiponectin gene polymorphisms with an elevated risk of diabetic peripheral neuropathy in type 2 diabetes patients. Journal of Diabetes and its Complications 201529 887-892. (doi:10.1016/j. jdiacomp.2015.06.008)

Received 12 October 2015

Revised version received 9 December 2015

Accepted 5 January 2016 